\title{
Wear of Fluorapatite Single Crystals: V. Influence of Environment on Frictional Behavior and Surface Failure
}

\author{
J. M. POWERS, K. C. LUDEMA, and R. G. CRAIG \\ School of Dentistry and College of Engineering, University of \\ Michigan, Ann Arbor, Michigan 48104, USA
}

The influence of environment on the frictional behavior and surface failure of fluorapatite single crystals was evaluated for sliding in air, water, and dimethylformamide. The coefficient of friction was independent of environment. The ductile-to-brittle transition, however, occurred at a lower load in water than in air.

In recent years, several investigations of the environment-sensitive mechanical behavior of certain crystalline, nonmetallic materials and glasses have been directed toward characterizing the wear behavior of these materials under conditions of sliding and drilling in active liquid environments. Buckley, ${ }^{1}$ for example, examined the change in plastic deformation and friction coefficient of single crystals of calcium fluoride with sliding in water and oleic acid. Westwood and Goldheim ${ }^{2}$ and Westwood, Goldheim, and Lye 3,4 have studied the environment-sensitive mechanical behavior of calcium fluoride and magnesium oxide.

The purpose of this study was to evaluate the influence of liquid environments on the frictional behavior and surface failure of the basal plane of fluorapatite single crystals.

\section{Materials and Methods}

A diamond hemisphere (360 micrometers

Based on a dissertation submitted in partial fulfillment of the requirements for the $\mathrm{PhD}$ degree in the Horace H. Rackham School of Graduate Studies at the University of Michigan, 1972.

This paper was presented, in part, at the 50th general session of the IADR in Las Vegas, Nevada, March 1972.

This investigation was supported, in part, by USPHS Training Grant DE-00181 from the National Institute of Dental Research, National Institutes of Health, Bethesda, Md.

Received for publication May 10, 1972. $[\mu \mathrm{m}]$ in diameter) a was slid ${ }^{5}$ across the basal surface of natural fluorapatite (FAP) single crystals. $^{b}$ Fifteen parallel, one-traversal scratches that resulted from sliding a normal load $(W)$ of 10 to $150 \mathrm{gm}$ in increments of $10 \mathrm{gm}$ were made on the basal plane of each of six crystals (FAP, 1 to 6 ) in the $x$ direction $[2 \overline{1} 10]$ in environments of air, water, and dimethylformamide (DMF), $\mathrm{c}$ another highly polar liquid. Tangential force and track width data were collected during each run. A scanning electron microscoped was used to study wear scars further. From failure classification data, we chose the maximum normal load above which a ductile mode of failure 6 (Class 1) was no longer observed, $\Omega$.

\section{Results}

Data for failure classification, track width, and tangential force are plotted as a function of normal load in Figures 1 and 2 for sliding on FAP1 in environments of water and DMF, respectively. Several curves calculated from models were compared with the measured data of these plots. A linear regression line was fit to the tangential force vs normal load data. ${ }^{7}$ The slope $(\beta)$ of this regression line and its standard error are indicated on the plot. The measured values of track width were compared qualitatively with values computed from an equation derived from a special case of Hertz's theory of contact between two elastic spheres; this approach was discussed previously. ${ }^{7}$ In addition to $\beta$ and $\Omega$, several other parameters ${ }^{7}$ were averaged over the set

\footnotetext{
a Wheel Trueing Tool Co., Detroit, Mich.

b Southwest Scientific Co., Hamilton, Mont.

c Matheson Coleman and Bell, Norwood, Ohio.

'JSM-U3, Japan Electron Optics Laboratory, Jap.
} 


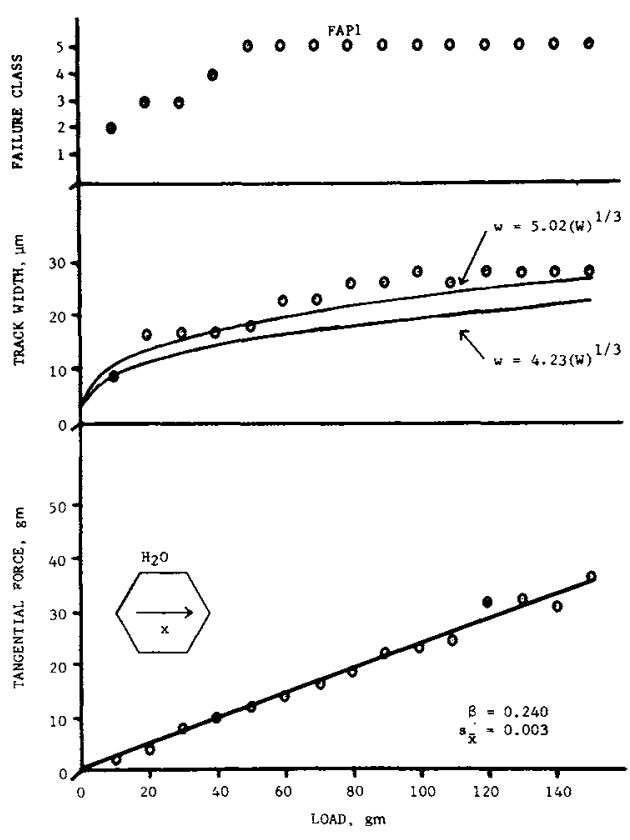

FIG 1.--Failure classification, track width, and tangential force vs normal load for FAPl in water in $x$ sliding direction.

of six crystals and are reported in the table for sliding in the $x$ direction in environments of air, water, and DMF.

Based on the assumption that the six fluorapatite crystals were representative of a sample of a larger population of crystals, a one-way analysis of variance was made for each of the parameters listed in the table. The factor of environment was tested at three levels (air, water, and DMF) with six replications per cell. The tangential force coefficient, $\beta$, in the $x$ sliding direction did not vary as a function of the environments tested at the 0.05 level of significance. An average value that could describe $\beta$ in the three environments was 0.234. An analysis of the variable $\Omega$, indicated that a difference existed among the three levels at the 0.05 level of significance, since $\mathrm{F}=3.97>F_{(2,15)}$ $=3.68$. Ranking of the means by the method of Duncan ${ }^{8}$ gave the following results:

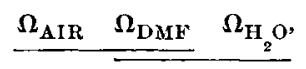

where the means that are underscored could not be distinguished from each other significantly.

Analysis of the variables, $w(\Omega), z(\Omega), d(\Omega)$. $p^{\prime}(\Omega), \tau_{\text {max }}(\Omega)$, and $\tau_{\text {boundary }}(\Omega)$, indicated that differences among the means at the three levels of environment existed at the 0.05 level of significance. Ranking of the means for each variable resulted in the following: air $=\mathrm{DMF}>\mathrm{H}_{2} \mathrm{O}$. An average coefficient of variation for these data was $27 \%$. Analysis of the variables, $F(\Omega)$ and $\sigma_{\mu}(\Omega)$, indicated that no difference could be distinguished among the three levels of environment at the 0.05 level of significance. The coefficients of variation for these data were 76 and $35 \%$, respectively. It was noted that $z(\Omega)$ was an order of magnitude larger than $d(\Omega)$ and that the variables describing stress based on the Hertzian model could be ranked in the following order for each environment: $p^{\prime}(\Omega)>\tau_{\max }(\Omega)>\sigma_{\mu}(\Omega)>$ $\tau_{\text {loundary }}(\Omega)$.

Scanning electron photomicrographs of wear scars that typify the failure observed in water are shown in Figure 3 for FAP2. For this particular crystal, evidence of tensile cracking (Class 2) was observed at a normal load of $10 \mathrm{gm}$. The center of the track appeared ductile. At a normal load of 20 gm, chipping in the center of the track was observed, although tensile cracks (Class 3) were evident along the edge of the wear

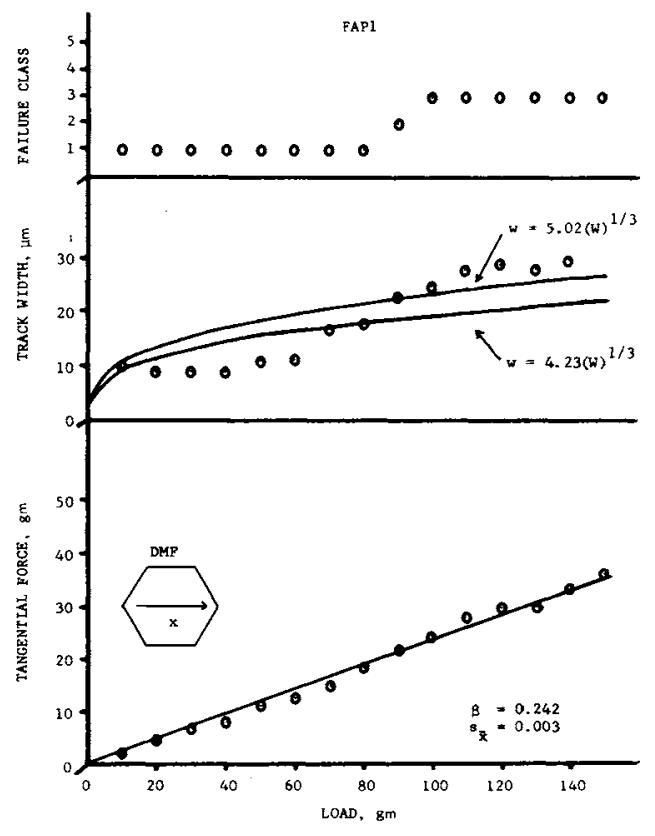

Fic 2.-Failure classification, track width, and tangential force vs normal load for FAPl in DMF in $x$ sliding direction. 


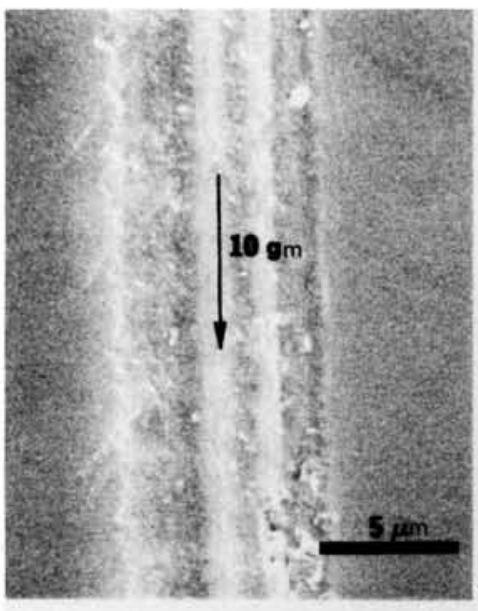

(a) $\mathrm{H}_{2} \mathrm{O}$

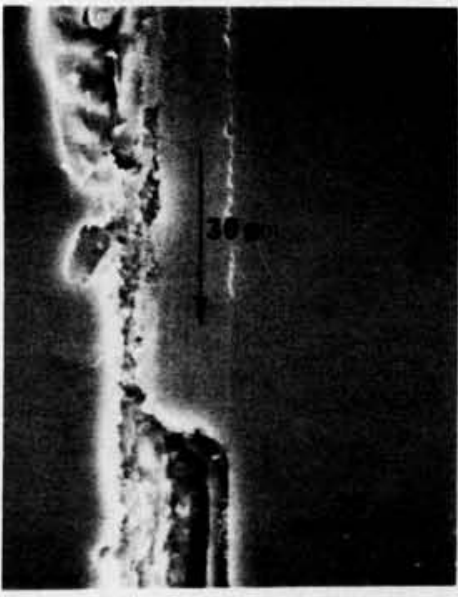

(c) $\mathrm{H}_{2} \mathrm{O}$

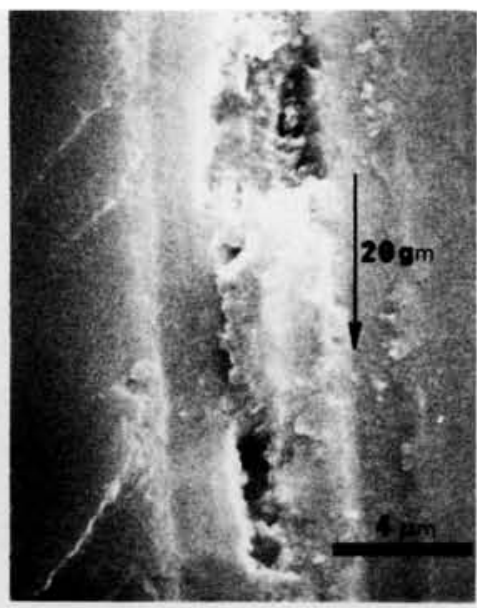

(b) $\mathrm{H}_{2} \mathrm{O}$

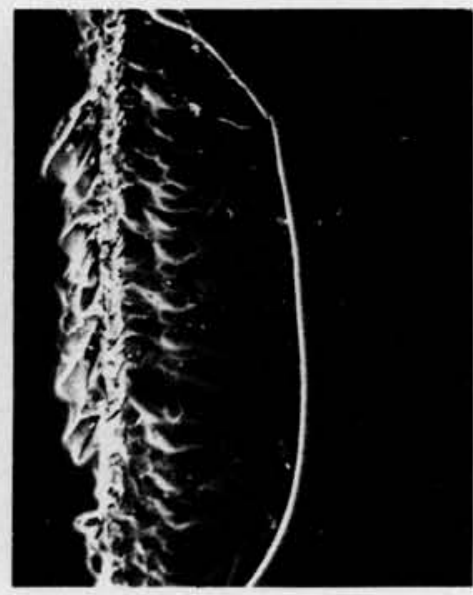

(d) $\mathrm{H}_{2} \mathrm{O}$

Fig 3.-Wear tracks formed in water environment. Arrow beside each track indicates direction of relative motion of slider with respect to surface of crystal. Value to right of arrow is normal load corresponding to particular wear scar. Scale for $c$ is $20 \mu \mathrm{m}$; force and scale for $d$ are $120 \mathrm{gm}$ and $67 \mu \mathrm{m}$. respectively.

scar. At a normal load of $30 \mathrm{gm}$, tensile cracking was no longer observed; rather, large fragments had chipped away from the center and along the edge of the wear scar. Ductile grooves in the center of the track were no longer observed. At higher loads, an unusual type of chevron pattern was observed at numerous intervals along the track, as shown for a $120 \mathrm{gm}$ load in Figure $3, d$. Lines of fracture associated with these chevron patterns were perpendicular to the wear scar, as shown in the aforementioned figure. Subsurface failure ${ }^{e}$ in the immediate vicinity of each wear scar was more prevalent with sliding in water than sliding in air or DMF. The areas of the wear tracks shown in the photomicrographs are representative of the particular wear scars as a whole.

- Subsurface failure of a crystal was characterized by observing the reflection of light during examination of the wear scars on the metallograph. This reflection of light was evaluated only qualitatively. 


\section{Discussion}

No differences were observed among the values of $\beta$ (Table) for sliding in the $x$ direction in environments of air (that is, adsorbed water), water, or DMF. In materials where slip is the predominant mechanism for relieving strain under sliding, the coefficient of friction is influenced by surfaceactive species. ${ }^{1}$ On single crystals of calcium fluoride, for example, sliding in oleic acid resulted in a decrease in the amount of plastic deformation and a corresponding de crease in the coefficient of friction. In addition, measurements of dislocation densities on the basal plane of FAP in the present study indicated no increase in density as the result of sliding. 9 These observations seem to indicate that slip is not the primary mechanism for relieving strain on the basal plane of FAP. Since a mechanism such as twinning has not been reported for fluorapatite (although it has been reported for chlorapatite ${ }^{10}$, it is suggested that the strain resulting from sliding is accommodated primarily by elastic deformation. Beyond a critical value of elastic strain, a minimal amount of slip (perhaps less than $10 \%$ ) may occur to relieve additional stress. Further stress results in the initiation and propagation of surface and subsurface cracks. Because of the small contribution of plowing friction, the independence of the coefficient of friction in the environments examined is not inconsistent.

Qualitative interpretation of the track width data suggested that there was probably little difference among the values measured for sliding in environments of air, water, and DMF. The fit of these values to the curve predicted by a Hertzian analysis was reasonably good. These observations support the hypothesis that only a minimal amount of plastic deformation occurs as a result of sliding on the basal plane of FAP.

The values of $\Omega$ for sliding in the $x$ direction in air, water, and DMF are compared in the table. Because of the scatter of data, it was difficult to observe differences statistically; however, a difference was observed between the means of $\Omega$ for sliding in air (adsorbed water) and water. Thus, the ductile-to-brittle transition occurs at a lower load for sliding in the $x$ direction in an environment of bulk water than in air. Values of $p^{\prime}(\Omega), \tau_{\max }(\Omega)$, and $\tau_{\text {houndury }}(\Omega)$ were lower for sliding in water than in air. However, no differences could be detected among the means of $\sigma_{\mu}(\Omega)$ in the various environments. This latter observation is thought to reflect the amount of scatter that existed in the $\beta$ and $\Omega$ data. It was apparent, however, that the mode of surface damage was influenced by sliding in water. Not only was surface cracking evident at lower loads, as indicated by $\Omega$, but the mechanism of surface failure appeared to have changed in the presence of bulk water. In general, damage as a result of sliding on the basal plane of FAP in water was considerably more severe when compared with damage resulting from sliding in air (adsorbed water) and DMF. No differences between sliding in air and DMF were observed.

Recently, Westwood, and Goldheim ${ }^{2}$ and Westwood. Goldheim, and Lye $^{3}$ have proposed a model to explain increases and decreases in surface hardness in the presence of surface-active species. The Westwood, Goldheim, and Lye model (WGL) ${ }^{2-4}$ assumes that the mobility of dislocations in ionic crystals is controlled predominantly by interactions with such point defects as vacancies and impurity atoms and that chemisorption on such materials involves, at least in part, electron transfer between near-surface point and line defects and the adsorbate. They suggest, essentially, that adsorption-induced changes in the electronic state of near-surface point defects and dislocations introduce variations in their mutual interactions.

Two possibilities to explain the decrease of $\Omega$ of FAP in water seem likely. The first is surface hardening. Buckley ${ }^{1}$ has observed such an effect in calcium fluoride with sliding in oleic acid. In this instance, the presence of oleic acid on calcium fluoride caused a decrease in deformability that was manifested by a decrease in the coefficient of friction and an increase in the amount of cracking under sliding. The second possibility is surface softening, with the restriction that fluorapatite under the experimental conditions be considered a notch-sensitive material. Both of these possibilities can be discussed most conveniently in terms of the WGL model.

Surface softening in the presence of bulk water requires an interaction between the polar water molecules or their dissociation products and charged, near-surface 
TABLE

Average Behavior of FAP Crystals as a Function of Environment

\begin{tabular}{|c|c|c|c|}
\hline \multirow[b]{2}{*}{ Property } & \multicolumn{3}{|c|}{ Environment } \\
\hline & Air & Water & DMF \\
\hline$\beta$ & $0.217(0.008) *$ & $0.241(0.017)$ & $0.245(0.009)$ \\
\hline $\begin{array}{c}\Omega \\
\left(g^{m}\right)\end{array}$ & $77(21)$ & $18(4)$ & $63(16)$ \\
\hline $\begin{array}{r}F(\Omega) \\
(\mathrm{gm})\end{array}$ & $17.2(5.2)$ & $4.7(1.3)$ & $16.1(4.4)$ \\
\hline $\begin{array}{c}w(\Omega) \\
(\mu \mathrm{m})\end{array}$ & $17.0(1.9)$ & $10.8(0.8)$ & $15.9(1.8)$ \\
\hline $\begin{array}{l}z(\Omega) \\
(\mu \mathrm{m})\end{array}$ & $4.1(0.5)$ & $2.6(0.2)$ & $3.8(0.4)$ \\
\hline $\begin{array}{r}d(\Omega) \\
(\mu \mathrm{m})\end{array}$ & $0.21(0.04)$ & $0.08(0.01)$ & $0.19(0.04)$ \\
\hline $\begin{array}{c}p^{\prime}(\Omega) \\
\left(\mathrm{kg} / \mathrm{mm}^{2}\right)\end{array}$ & $429(48)$ & $274(20)$ & $402(46)$ \\
\hline $\begin{array}{c}\tau_{\max }(\Omega) \\
\left(\mathrm{kg} / \mathrm{mm}^{2}\right)\end{array}$ & $135(15)$ & $86.1(6.4)$ & $126(14)$ \\
\hline $\begin{array}{c}\tau_{\text {boundarg }}(\Omega) \\
\left(\mathrm{kg} / \mathrm{mm}^{2}\right)\end{array}$ & $62.9(7.1)$ & $40.1(3.0)$ & $58.9(6.8)$ \\
\hline $\begin{array}{c}\sigma_{\mu}(\Omega) \\
\left(\mathrm{kg} / \mathrm{mm}^{2}\right)\end{array}$ & $94.2(13.1)$ & $67.3(9.5)$ & $100(14)$ \\
\hline
\end{tabular}

- Standard error of the mean is in parentheses.

species such that surface plasticity is improved. The increased cracking in water, however, requires that FAP be notch-sensitive and that the dislocation process lead to crack initiation through the formation of microcracks. However, microcrack formation by means of a dislocation process requires multiple slip systems, 11 a criterion that is apparently not met in FAP.

Surface hardening in the presence of bulk water also requires an interaction between the polar water molecules or their dissociation products and charged, near-surface species. This interaction would result in the pinning of dislocations, thereby reducing the ability of the lattice to accommodate strain by slip and effectively lowering the fracture stress. This model is consistent with the behavior observed for sliding on the basal plane of FAP. Since the accommodation of strain by slip in FAP is a limited process in air, further limitations on dislocation movement would lead to crack initiation. Only a slight reduction in the coefficient of friction is expected, since the plowing component of friction was small initially. Furthermore, examination of the fracture surfaces that resulted from sliding in water indicated that less grooving occurred at low loads. At higher loads the entire track chipped away in water; in air, a ductile track was bordered by a chevron pattern.

The possibility of interactions according to the WGL model occurring in fluorapatite for sliding in various environments is reasonable. In fluorapatite the fluorine is located at the center of the calcium triangle. ${ }^{12}$ The channels formed by these calcium triangles are fairly large and are supported by the remainder of the structure, such that they provide easy diffusion paths. ${ }^{13}$ Surface-active agents could influence the position of the near-surface $F^{-}$ions relative to the calcium triangles, thereby modifying the properties of the surface. However, these surface-active agents must be present in a liquid or insulating environment so that surface charge effects would not be dissipated in air; this explains the differences in behavior observed for fluorapatite in bulk water and in air (adsorbed water).

In all cases observed for sliding in various environments, as well as in the $x$ and $y$ sliding directions, the magnitude of $\sigma_{\mu}(\Omega)$ 
was greater than that of $\tau_{\text {boundary }}(\Omega)$. The mode of surface failure might change if $\sigma_{\mu}$ were less than $\tau_{\text {boundary }}$. For $\sigma_{\mu}$ to be less than $\tau_{\text {boundary }}$, the value of $\beta$ must be lowered by 50 to $100 \%$. An attempt to reduce $\beta$ by sliding in a lubricant failed. ${ }^{9}$ This failure, however, does support the argument that the deformation component of friction is small.

\section{Conclusions}

The influence of environment on the frictional behavior and surface failure of the basal plane of natural fluorapatite single crystals was evaluated in the [2/ī0] direction.

No differences could be detected among the values of $\beta$ for sliding in air (adsorbed water), water, and DMF. Qualitative interpretation of the track width suggested the major mechanisms for the accommodation of strain as a result of sliding were elastic deformation and cracking. No differences in track width were detected for the environments studied. The ductile-to-brittle transition occurred at a lower load for sliding in water than in air or DMF. The mechanism of failure appeared to be different in water. A model based on surface hardening was proposed to explain the unusual mode of failure observed in bulk water.

\section{References}

1. Buckley, D.H.: Effect of Surface Active Media on Friction, Deformation and Frac. ture of Calcium Fluoride, Washington, DC: NASA TN D-5580, 1969.

2. Westwood, A.R.C., and GOLDHEIM, D.L.: Occurrence and Mechanism of Rebinder Ef- fects in $\mathrm{CaF}_{2}, J$ Appl Phys 39: 3401-3405, 1968.

3. Westwoon, A.R.G.; GoldheIM, D.L.; and LYE, R.G.: Rebinder Effects in MgO, Philosophical Mag 16: 505-519, 1967.

4. Westwoon, A.R.C.; GoLdHEIM, D.L.; and LYE, R.G.: Further Observations on Rebinder Effects in $\mathrm{MgO}$, Philosophical Mag 17: $951-959,1968$.

5. Powers, J.M., and Craig, R.G.: Wear of Fluorapatite Single Crystals: I. A Method for Quantitative Evaluation of Wear, $J$ Dent Res 51: 168-176, 1972.

6. Powers, J.M., and Craig, R.G.: Wear of Fluroapatite Single Crystals. III. Classification of Surface Failure, J Dent Res 51:611$618,1972$.

7. Powers, J.M.; Ludema, K.C.; and Craic, R.G.: Wear of Fluorapatite Single Crystals: IV. Influence of Sliding Direction on Frictional Behavior and Surface Failure, $J$ Dent Res 52: 1019-1025, 1973.

8. Dungan, D.B.: Multiple Range and Multiple F-Test, Biometrics 11: 1-42, 1955.

9. Powers, J.M.: The Influence of Crystallographic Direction and Environment on the Ductile to Brittle Transition of Fluorapatite Single Crystals Under Sliding, dissertation, University of Michigan, Ann Arbor, 1972.

10. Prener, J.S.: The Growth and Crystallographic Properties of Calcium Fluor- and Chlorapatite Crystals, J Electrochem Soc I14: 77-83, 1967.

11. Clarke, F.J.P.; Sambell, R.A.J.; and TatTERSALL, H.G.: Mechanisms of Microcrack Growth and Magnesium Oxide Crystals, Philosophical Mag 7: 393-413, 1962.

12. Naray-Szabo, S.: The Structure of Apatite (CaF) $\mathrm{Ca}_{4}\left(\mathrm{PO}_{4}\right)_{3}, Z$ Kristollogr Kristallgeom 75: 387-398, 1930.

13. Younc, R.A., and Elliott, J.C.: Atomiccale Bases for Several Properties of Apatites, Arch Oral Biol 11: 699-707, 1966. 